\title{
Recent advances in cannabinoid biochemistry and biotechnology
}

\author{
Futoshi Taura $^{\mathrm{a}, *}$, Ryosuke Tanaya ${ }^{\mathrm{a}}$, Supaart Sirikantaramas ${ }^{\mathrm{b}, *}$ \\ a Graduate School of Medicine and Pharmaceutical Sciences for Research, University of Toyama, Sugitani, \\ Toyama 930-0194 Japan \\ b Molecular Crop Research Unit, Department of Biochemistry, Faculty of Science, Chulalongkorn \\ University, Bangkok 10330 Thailand
}

*Corresponding authors, e-mail: taura@pha.u-toyama.ac.jp, supaart.s@chula.ac.th

Received 13 Oct 2019

Accepted 18 Oct 2019

\begin{abstract}
Cannabinoids, produced only in Cannabis sativa, are meroterpenoid secondary metabolites composed of monoterpene and polyketide moieties. Numerous cannabinoids have been isolated, and their pharmacological studies and clinical trials have been extensively conducted. Consequently, in recent years, certain formulations containing $\Delta^{9}$-tetrahydrocannabinol, the psychoactive principle of marijuana, and its isomer cannabidiol have been approved as prescribed medicines for various refractory diseases in about 30 countries. In addition, recent pioneering studies using a set of biosynthetic genes have enabled synthetic biology and synthetic biochemistry approaches to access plant-derived cannabinoids and new-to-nature analogues without the need for plant breeding. This review introduces the history of cannabinoid biosynthetic studies, and focuses on the biotechnological applications of biosynthetic enzymes.
\end{abstract}

KEYWORDS: Cannabis sativa, cannabinoid, biosynthesis, synthetic biology, synthetic biochemistry

\section{Cannabis and cannabinoids}

Cannabis sativa L., an annual herb belonging to Cannabaceae, known as marijuana or hemp, has been cultivated for medicinal, fibre industrial and recreational purposes around the world for thousands of years ${ }^{1,2}$. Cannabis plants produce unique specialized metabolites called cannabinoids, which consist of polyketide and monoterpene substructures (Fig. 1). Hitherto, more than 100 cannabinoids have been isolated from marijuana and hemp, and their chemical and pharmacological properties have been intensively investigated ${ }^{1,2}$. Among these metabolites, $\Delta^{9}$-tetrahydrocannabinol (THC), identified as the psychoactive cannabinoid ${ }^{3}$, has engaged considerable attention because this cannabinoid has valuable therapeutic potentials, including analgesic, anticonvulsant, antiemetic and appetitestimulating properties ${ }^{4}$.

Cannabidiol (CBD), a THC isomer having a different ring system, has also become very notable in recent years as a potent antiseizure drug to treat intractable childhood epilepsy ${ }^{4}$. It is also known that $\mathrm{CBD}$ can reduce the unfavorable psychotropic effects of THC. Thus, Nabiximols (Sativex ${ }^{\circledR}$ ), a Cannabis-based oral spray consisting of THC and $\mathrm{CBD}$ in a 1:1 $(\mathrm{w} / \mathrm{w})$ ratio, has been approved in
Canada and several European countries to alleviate various symptoms including pain and spasms associated with multiple sclerosis, whereas the Food and Drug Administration has recently approved the CBD-based medicine Epidiolex ${ }^{\circledR}$ to treat rare and severe childhood epilepsy, such as Dravet and LennoxGastaut syndromes ${ }^{5,6}$.

It has been postulated that not only the terpene ring differences, as seen between $\Delta^{9}$-THC and CBD, but also the alkyl chain structures in cannabinoids could be an important pharmacophore to modulate their pharmacological activities ${ }^{7,8}$. For example, $\Delta^{9}$-tetrahydrocannabivarin (THCV), which has a propyl side chain instead of a pentyl group, antagonizes the mammalian cannabinoid receptors CB1 and $\mathrm{CB} 2^{7,8}$. It is known that metabolic diseaserelated parameters, such as serum triglyceride, HDL cholesterol and adiponectin concentrations, are regulated by cannabinoid receptor antagonists. Actually, recent preclinical studies have suggested that THCV could be a potential treatment for obesity, type 2 diabetes and related metabolic disorders ${ }^{9}$. Thus, plant cannabinoids have regained interest as potential therapeutic agents, making cannabinoid biochemistry, which would lead to biotechnological production of active ingredients, a more and more important research field in recent years. 


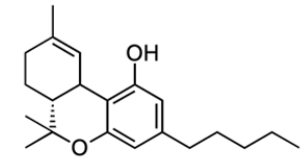

$\Delta^{9}$-Tetrahydrocannabinol (THC)

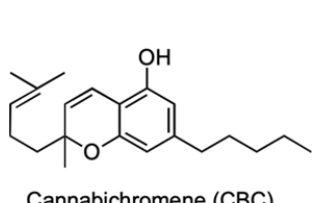

Cannabichromene (CBC)

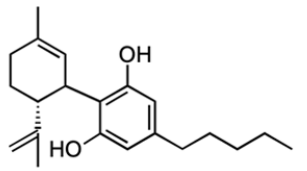

Cannabidiol (CBD)

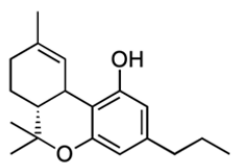

$\Delta^{9}$-Tetrahydrocannabivarin (THCV)
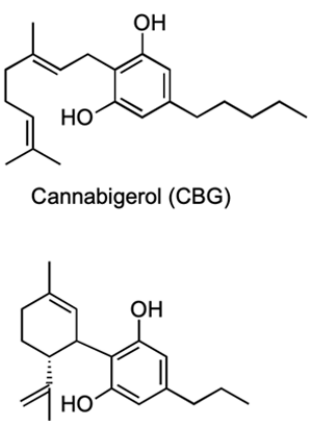

Cannabidivarin (CBDV)

Fig. 1 Structures of representative cannabinoids. Their generally accepted abbreviations are presented in parentheses.

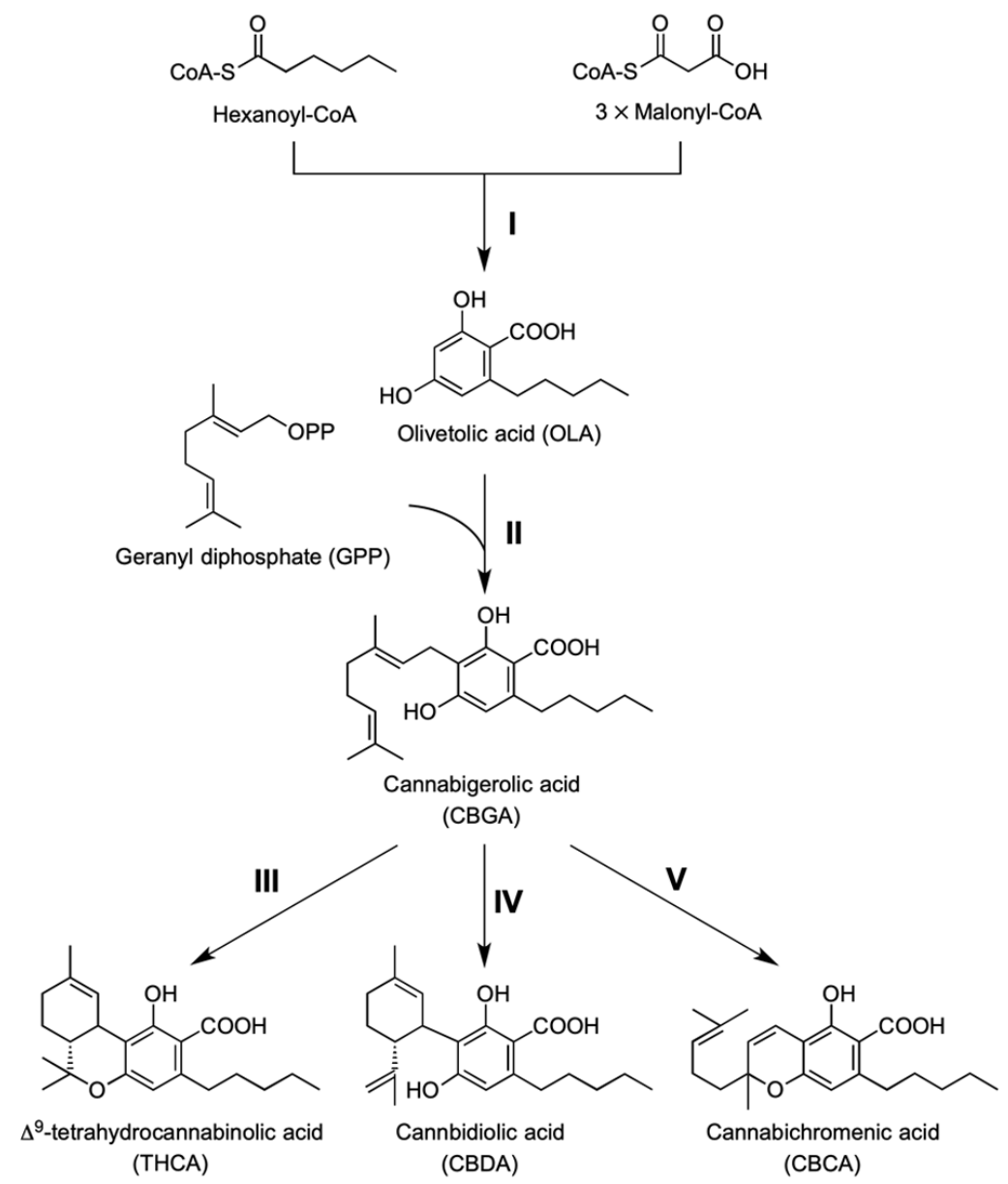

Fig. 2 Biosynthetic pathway of cannabinoids. The biosynthetic enzymes catalysing respective steps are as follows. I, tetraketide synthase and olivetolic acid cyclase; II, CsPT4, a prenyltransferase; III, THCA synthase; IV, CBDA synthase; $\mathrm{V}$, CBCA synthase.

In fresh Cannabis plants, cannabinoids are biosynthesized and accumulated as cannabinoid acids, such as $\Delta^{9}$-tetrahydrocannabinolic acid
(THCA) and cannabidiolic acid (CBDA), and nonenzymatically decarboxylized into their neutral forms during storage and smoking ${ }^{10}$. As shown 
in Fig. 2, cannabinoid biosynthesis is composed of three catalytic steps: (i) polyketide formation, (ii) prenylation, and (iii) oxidative cyclization ${ }^{10}$. Modern studies on cannabinoid biosynthesis was launched a quarter of century ago when THCA synthase was identified in the young leaves of C. sativa ${ }^{11}$. The early biochemical studies made gradual progress, but recent integrated omics-based approaches have greatly accelerated the process to almost completely identify the enzymes specialized to this pathway, providing the motivation for scientists to elaborate on cannabinoid biotechnology. This review summarizes the unique features of these biosynthetic enzymes, and outlines the potential and difficulties in their biotechnological applications to produce active cannabinoid metabolites.

\section{Olivetolic acid (OLA) biosynthesis catalyzed by a novel polyketide cyclase}

The first committed step in cannabinoid biosynthesis is polyketide (olivetolic acid, OLA) formation from hexanoyl-CoA and three molecules of malonyl-CoA. This step is catalyzed by tetraketide synthase (TKS) and olivetolic acid cyclase (OAC), as established by omics-based studies ${ }^{12,13}$ (Fig. 3). The sequential co-action mechanism of polyketide formation is unique to OLA biosynthesis, because known plant polyketide synthases (PKSs) can complete their reactions to form aromatic polyketides ${ }^{14}$. In contrast, Cannabis PKS is called TKS, because it releases the tetra- $\beta$-ketide-CoA intermediate without cyclization into aromatics ${ }^{13}$. Alternatively, OAC catalyzes $\mathrm{C} 2$ to $\mathrm{C} 7$ aldol condensation of the linear intermediate supplied by TKS to provide OLA as a cannabinoid precursor ${ }^{13}$. The only polyketide cyclase in the plant kingdom, OAC is the primary factor to pave the unique biosynthetic route leading to cannabinoids. In addition, an acyl-activating enzyme named AAE1 that specifically produces hexanoyl-CoA, the starter substrate for OLA biosynthesis (Fig. 3), has been identified ${ }^{15}$.

The identification of OAC and AAE1, along with TKS, provided the strategy for the biotechnological production of OLA in microorganisms. An efficient synthetic pathway of OLA by heterologous expression of these three enzymes in Escherichia coli, which was engineered to robustly supply CoA-linked substrates from sugar, was recently established ${ }^{16}$. A similar strategy was subsequently employed to develop cannabinoid-producing yeast strains ${ }^{17}$. The recently reported crystal structure of OAC contained a hydrophobic pentyl binding pocket that accommodates substrates with a pentyl side chain ${ }^{18}$. The ra- tional modification of the OLA active site, especially on the pentyl-binding pocket, might expand the catalytic repertories to provide novel cannabinoid precursors.

\section{Aromatic prenyltransferase: the uncertain-link in the pathway}

The next prenylation step produces cannabigerolic acid (CBGA), an important cannabinoid as the central precursor for a number of major metabolites, such as THCA and CBDA. The prenyltransferase (PT) activity, which produces CBGA via condensation between OLA and geranyl pyrophosphate (GPP), was identified in the young leaves of Cannabis plants about 20 years ago ${ }^{19}$. It was demonstrated that the monoterpene moieties in cannabinoids are derived from the methylerythritol 4-phosphate (MEP) pathway ${ }^{20}$. Because the MEP pathway generally operates in the plastids of higher plants, plastid-localized PT was expected to participate in CBGA biosynthesis. In fact, a cDNA encoding an aromatic PT (CsPT1) with a sequence homology to plastid-localized plant PTs was identified ${ }^{21}$, and CsPT1 has since long been accepted as a PT in the cannabinoid pathway, although its biochemical properties were unusual for a PT involved in plant specialized metabolism. For example, the recombinant CsPT1 showed non-specific substrate specificity in accepting various aromatic substrates with higher efficiencies than that for OLA ${ }^{21}$. Thus far, CsPT1, the uncertain link in the pathway, has not been employed as a biocatalyst for CBGA production.

Alternatively, researchers became interested in the utilization of bacterial PTs, such as NphB (formerly Orf2) from the Streptomyces sp. strain CL19022, since it also has promiscuous substrate preferences, but unlike plant enzymes, NphB is a soluble protein that is suitable for bacterial overexpression and structure-function studies. As a proof of concept, heterologous expression of $\mathrm{NphB}$ together with THCA synthase was performed in the methylotrophic yeast Komagataella phaffi ${ }^{23}$. Incubation of the recombinant strain with OLA and GPP produced detectable amounts of CBGA and THCA, suggesting that the pathway engineering worked functionally in the yeast. However, not only the efficiency, but also the regioselectivity of NphB turned out to be an obstacle, as the major bioconversion product was characterized to be 2-O-geranyl OLA (Fig. 4A), and the CBGA production level was unsatisfactory ${ }^{23}$. To overcome these problems, two research groups conducted structure-based functional 


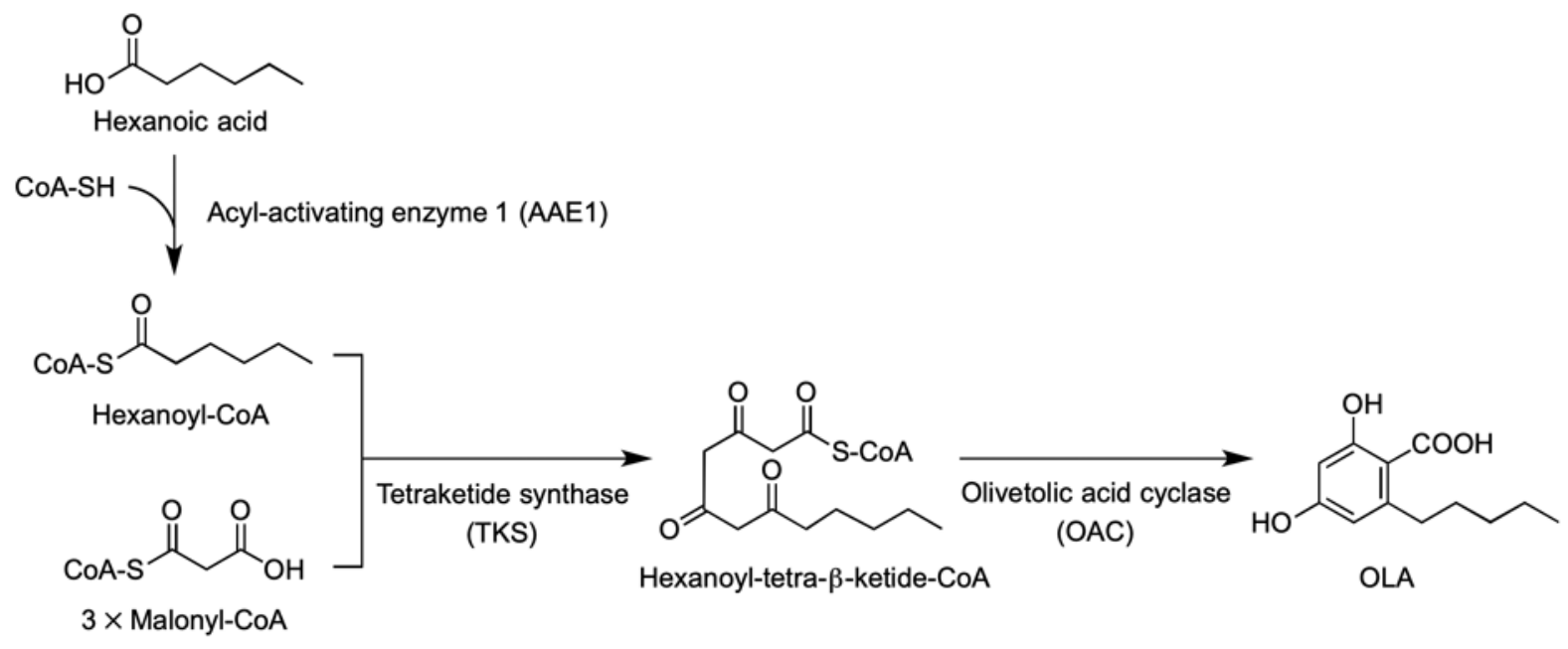

Fig. 3 Biosynthesis of OLA via co-action of TKS and OAC. An acyl-activating enzyme AAE1 supplies the starter molecule hexanoyl-CoA.<smiles>CCCCCc1cc(O)cc(O)c1C(=O)O</smiles><smiles>[B]C(=O)c1cc(O)cc(O)c1C(=O)O</smiles>

Fig. 4 Enzymatic formation of CBGA. The reactions catalyzed by (A) NphB and (B) CsPT4.

redesign of the NphB active site to improve the catalytic activity and regioselectivity ${ }^{24,25}$, with one of the redesigned NphB isoforms achieving these objectives for the synthetic biochemistry of cannabinoids as described below.

On the other hand, very recently, the genuine PT (CsPT4) in the cannabinoid pathway has been identified ${ }^{17}$. It shares $60 \%$ sequence identity with CsPT1 and specifically and effectively produced $\mathrm{CBGA}^{17}$ (Fig. 4B). This identification of the missinglink enzyme was a great progress to realize the synthetic biology of cannabinoids as described in the last section.
Cannabinoid-synthases: novel FAD-linked oxidases that catalyze the oxidative cyclization of CBGA

The final steps of cannabinoid biosynthesis are the stereoselective oxidative cyclization of the monoterpene moiety of CBGA to form bicyclic or tricyclic cannabinoids, including THCA and CBDA. These two compounds primarily occur in the trichomes on flowers and leaves, and are hallmark metabolites of the Cannabis chemical phenotypes called drug-type (marijuana) and fiber-type (hemp), respectively ${ }^{1,2}$. Female flowers of the pure drug-type strain contain more than 20\% THCA (by dry weight), whereas the corresponding tissues of the fiber type produce a comparable amount of $\mathrm{CBDA}^{26}$. In contrast, 
cannbichromenic acid (CBCA) is distributed in both the drug-type and fiber-type plants as a minor constituent $^{1,2}$.

The cannabinoid synthases, THCA synthase and CBDA synthase, belong to the vanillyl alcohol oxidase (VAO)-type having a covalently attached FAD molecule as a coenzyme ${ }^{27,28}$. The VAO flavoprotein family includes diverse plant enzymes that function in secondary metabolism, such as a berberine bridge enzyme involved in benzylisoquinoline alkaloid biosynthesis and precondylocarpine acetate synthase that participates in vinca alkaloid biosynthesis ${ }^{29,30}$. Cannabinoid synthases, along with the recently identified daurichromenic acid synthase in Rhododendron dauricum L. ${ }^{31}$, are novel members of FAD oxidases that are specialized for meroterpenoid biosynthesis.

THCA synthase is the first identified and cloned cannabinoid synthase that produces THCA, the acidic precursor of $\mathrm{THC}^{11,27}$. The work of our group focused on the expression in eukaryotic host organisms, such as yeast and insect cell systems ${ }^{27,32}$, biochemical characterization, biotechnological studies with THCA synthase as a model cannabinoid synthase, and the X-ray crystal structure analysis to demonstrate the enzyme reaction mechanism (Fig. 5) ${ }^{27,33}$. With respect to the mechanism, enzyme-bound FAD abstracts a hydride ion from the benzyl position of CBGA, and Tyr484 removes a proton from the phenolic hydroxyl group. Then, the ionic intermediate is cyclized stereoselectively into THCA, whereas the reduced FAD is re-oxidized using molecular oxygen as the final electron acceptor to release hydrogen peroxide as a by-product (equimolar to THCA). Because cannabinoids as well as hydrogen peroxide are toxic to plant cells, this reaction takes place in the extracellular compartments, such as the secretory cavity of glandular trichomes ${ }^{34,35}$. Thus, it is reasonable that THCA synthase is a secreted biosynthetic enzyme ${ }^{34}$.

The CBDA synthase has been identified and cloned as the second cannabinoid synthase that determines the chemical phenotype of $C$. sativa (fiber type or hemp) ${ }^{28,36}$. The primary structure of CBDA synthase shares a very high (about 84\%) amino acid identity with THCA synthase ${ }^{28}$. As illustrated in Fig. 6, this enzyme stereoselectively synthesizes CBDA from CBGA via FAD and an oxygen dependent mechanism, which is closely similar to that of THCA synthase $^{28}$. The significant difference between these reactions is seen in the proton transfer step; CBDA synthase abstracts a proton from the terminal methyl group of CBGA instead of from the hydroxyl group as observed in the THCA synthase reaction (Fig. 6). Based on their sequence and reaction similarities, it was preconceived that subtle active site differences in these cannabinoid synthases would regulate oxidative cyclization reactions to form different ring systems ${ }^{28}$.

In addition, a recent study demonstrated the multiple product forming-nature of cannabinoid synthases, where THCA synthase produced CBDA and $\mathrm{CBCA}$ as minor reaction products, whereas, similarly, CBDA synthase also synthesizes small amounts of THCA and CBCA as alternative cyclization products ${ }^{37}$. A series of site-directed mutant cannabinoid synthases have also been prepared to improve the catalytic activities, and to modify product specificities ${ }^{37}$, and as a typical result, demonstrated that the CBDA synthase variant A414V not only showed an increased catalytic activity, but also exhibited a 19-fold increased production of THCA as a byproduct, suggesting that the A414 residue in CBDA synthase regulates both its activity and specificity ${ }^{37}$.

Judging from the in vitro multiple productformation abilities of cannabinoid synthases, it is highly probable that CBCA is generated as a side product of THCA synthase and CBDA synthase in planta. On the other hand, the high-quality genome sequence of Cannabis has assisted in the identification of a genomic DNA coding for an enzyme that selectively produces $\mathrm{CBCA}^{38}$. This genomic CBCA synthase shares $93 \%$ sequence identity with THCA synthase, supporting the fact that slight amino acid substitutions alter the cyclization specificities. Nevertheless, further studies should be performed to confirm whether this genomic clone for $\mathrm{CBCA}$ synthase is actually expressed, and functions, in Cannabis plants.

\section{Advanced biotechnology for the production of cannabinoids and related compounds}

As described in the earlier sections, most of the biosynthetic enzymes specialized to the cannabinoid pathway have been identified and cloned, making it possible to develop alternative strategies for cannabinoid production.

An important advance was the establishment of an efficient bioconversion strategy to produce THCA upon feeding of CBGA using the methylotrophic yeast $K$. phaffii ${ }^{39}$. To intracellularly express the recombinant THCA synthase for a whole cell bioconversion, the vacuolar-targeting signal of proteinase A was fused with the mature THCA synthase $^{39}$. Consequently, about $1 \mathrm{mM}(36 \mathrm{mg} / \mathrm{l})$ of 


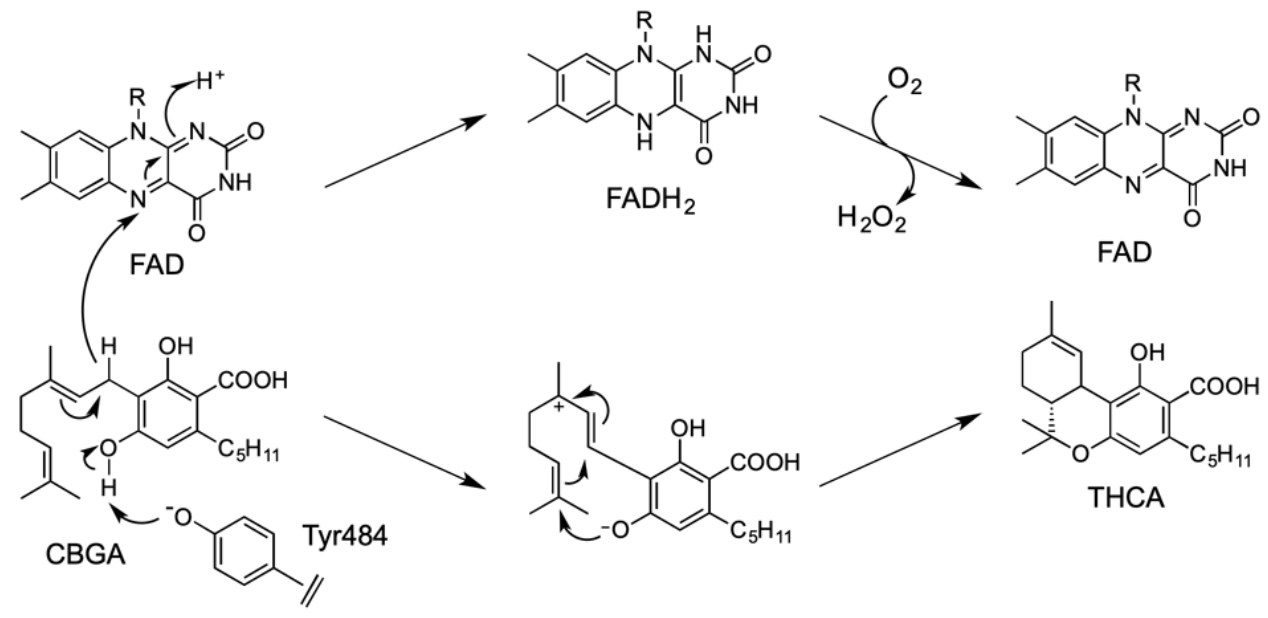

Fig. 5 Reaction mechanism of THCA synthase. R, the rest of FAD molecule.

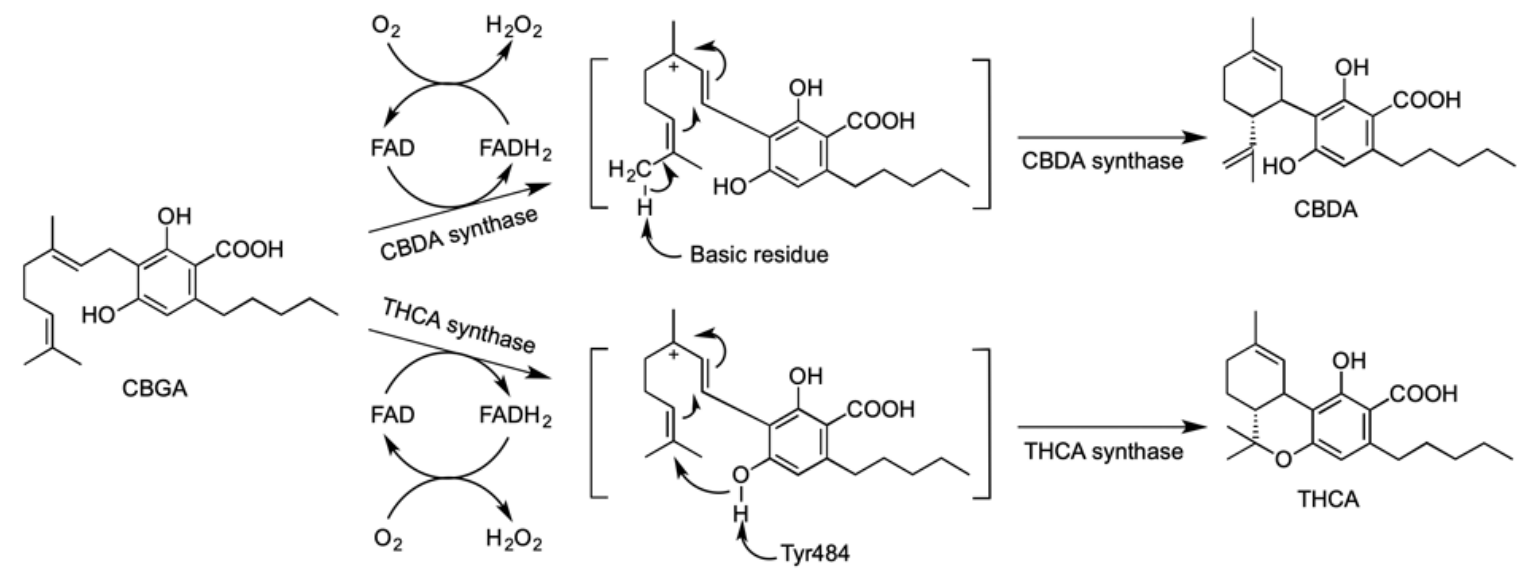

Fig. 6 Comparison of reaction schemes between CBDA synthase and THCA synthase.

THCA was produced in the cell cultures ${ }^{39}$. The vacuolar targeting of THCA synthase might be very reasonable for the production of THCA, because the vacuole seems to be a suitable site to sequester THCA, which otherwise could be toxic to the yeast. In addition, catalase, which is localized in yeast vacuole, would effectively detoxify the hydrogen peroxide by-product of THCA synthase ${ }^{40}$. This vacuolar-targeting strategy was later employed for the synthetic biology of cannabinoids in the yeast Saccharomyces cerevisiae ${ }^{17}$. In the early 2019 , two pioneering studies dealing with very different strategies for cannabinoid synthesis were published consecutively; one was about in vitro enzymatic total synthesis, whereas the other accomplished in vivo complete biosynthesis ${ }^{17,25}$. It should be noted that these studies for the first time enabled to fully access cannabinoids based on advanced biotechnological approaches.

The in vitro strategy was named synthetic biochemistry ${ }^{25}$, to distinguish it from synthetic biology where genetic manipulation is performed in vivo. To realize this novel concept, they not only expressed specialized biosynthetic enzymes, but also collected more than 20 enzymes required for supply of the expensive precursor molecule GPP from glucose. They constructed artificial glucose breakdown, acetylCoA synthesis, and isoprenoid-producing pathways in vitro by the combinatorial application of multiorganism-derived enzymes ${ }^{25}$. Furthermore, they utilized the aforementioned NphB mutant with improved reaction regioselectivity ${ }^{25}$ to specifically synthesize CBGA. In the presence of OLA, the reaction mixture produced more than $1 \mathrm{~g} / 1$ of CBGA, which 
was recovered in the nonane overlay ${ }^{25}$. Then, the obtained nonane fraction containing CBGA was just overlaid on an aqueous solution containing the recombinant CBDA synthase. This simple two-layer reaction system gradually but constantly synthesized CBDA with a final conversion rate of $25 \%$ after $4 \mathrm{~d}$ incubation ${ }^{25}$. Although the recombinant version of CBDA synthase is reportedly less active than that of the native enzyme ${ }^{28}$, it might be stable enough for a long reaction time. Consequently, a novel cell-free platform for cannabinoid production from inexpensive glucose and OLA has been successfully established $^{25}$.

Synthetic biochemistry is a somewhat complicated strategy because so many enzymes have to be prepared in a sufficient amount. However, this methodology overcomes the common problems in the trials of synthetic biology of natural products which may be harmful to host organisms ${ }^{25}$. Such problems are especially true for defence-related toxic compounds, including cannabinoids, which could be toxic both in prokaryotic and eukaryotic cell cultures ${ }^{34,41}$. Thus, the synthetic biochemistry approach might be superior to synthetic biology, especially in the case of producing toxic natural products. Needless to say, enzymatic synthesis is more environment-friendly than organic synthesis as it requires less toxic reagents and organic solvents.

Although synthetic biochemistry is now possible, synthetic biology is still invaluable because one can easily obtain natural products by a costeffective microbial fermentation once an elite production strain is established. Actually, S. cerevisiae strains that can biosynthesize cannabinoids and analogues from simple molecules, such as sugar and hexanoate, have been developed ${ }^{17}$. This study compiled the fruits of years of studies on cannabinoid biosynthesis, biotechnology, and various strategies for pathway engineering in microbial cell factories.

To this end, the TKS and OAC genes were first integrated into the yeast genome to perform preliminary OLA synthesis ${ }^{17}$. Then, the co-expression of the acyl-activating enzyme AAE1 to promote OLA production upon feeding of hexanoate was tested. Alternatively, a multi-organism-derived hexanoylCoA producing pathway was reconstituted in the yeast for de novo OLA synthesis. Thereafter, TKS, $\mathrm{OAC}$, and AAE1 were integrated into the CsPT4 strain and further reconstructed the hexanoyl-CoA pathway for de novo cannabinoid biosynthetic platform by introducing genes encoding either THCA synthase or CBDA synthase. Using these strains, they finally established complete biosynthesis of cannabinoids. The hexanoyl-CoA-producing yeast harboring THCA synthase synthesized $2.3 \mathrm{mg} / 1$ THCA after fermentation in the presence of galactose, whereas the corresponding strain expressing CBDA synthase yielded $4.2 \mu \mathrm{g} / 1 \mathrm{CBDA}^{17}$. The titer of THCA was improved to $8.0 \mathrm{mg} / 1$ by optimizing the copy numbers of the respective biosynthetic genes. However, the productivity of CBDA was limited, presumably due to the weak catalytic activity of the recombinant CBDA synthase ${ }^{28}$. It was also noted that these transgenic yeasts could produce propyl cannabinoids, such as THCVA and cannabidivarinic acid (CBDVA), respectively, from the butyrylCoA that was supplied as an intermediate of the hexanoyl-CoA pathway. These THC- and CBD-type propyl cannabinoids have recently become regarded as candidate molecules for various medicinal applications $^{9,42}$.

To expand the accessible chemical space of molecules, a precursor-directed biosynthesis by feeding various fatty acids to yeast cultures was conducted, leading to the production of a series of novel THCA analogues with side chains of variable length, branches, and degree of saturation ${ }^{17}$. Hence, it is evident that the acyl-activating enzyme AAE1 and several cannabinoid pathway-specific enzymes have a promiscuous nature to accept and modulate several different precursors. Furthermore, this biosynthetic strategy also afforded analogues harboring a terminal alkyne moiety, which could be derivatized by click chemistry to synthesize biotinylated cannabinoid derivatives ${ }^{17}$.

It is needless to say, these novel cannabinoid analogues and derivatives would be promising medicinal resources to explore various biological activities as well as being useful chemical probes for cannabinoid related bio-analytical chemistry. Thus, synthetic biology actually opened the gate to get cannabinoids and new-to-nature analogues in our hands, even though fine-tuning of the system should be conducted to improve the productivity for industrial and commercial purposes.

\section{Concluding remarks}

The identification of the complete cannabinoid biosynthetic genes in Cannabis plants has been a long-awaited breakthrough since the psychoactive THC was first identified in $1964^{3}$. This discovery represents a big step forward in the biotechnological productions of cannabinoids that have been published in the last few years. With the availability of a high-resolution genome map of Cannabis ${ }^{40}$, we 
are expecting more novel genes related to a diverse array of cannabinoids to be discovered and molecular markers associated with unique chemo-types to be designed. As we enter the post-genomic era of Cannabis plants together with the advancement of synthetic biology and biochemistry, novel elite Cannabis strains can be created and new-to-nature cannabinoid analogues can be produced in the plant or in/with alternative organisms/platforms. An urgent need to further understand the detailed mechanisms of action of cannabinoids and their novel analogues in human is required for opening up new potential therapeutic approaches in treating various diseases.

Acknowledgements: The research in our laboratories was partially supported by a Grant-in-Aid for the Cooperative Research Project from Institute of Natural Medicine, University of Toyama in 2018 (to FT) and grants from the Ratchadapisek Sompoch Endowment Fund (2019), Chulalongkorn University: Chula Cannabis and its Natural Substances Cluster and GRU 6203023003-1 (to SS).

\section{REFERENCES}

1. Andre CM, Hausman JF, Guerriero G (2016) Cannabis sativa: the plant of the thousand and one molecules. Front Plant Sci 7, ID 19.

2. Bonini SA, Premoli M, Tambaro S, Kumar A, Maccarinelli G, Memo M, Mastinu A (2018) Cannabis sativa: a comprehensive ethnopharmacological review of a medicinal plant with a long history. $J$ Ethnopharmacol 227, 300-315.

3. Gaoni Y, Mechoulam R (1964) Isolation, structure, and partial synthesis of an active constituent of hashish. J Am Chem Soc 86, 1646-1647.

4. Russo E, Guy GW (2006) A tale of two cannabinoids: the therapeutic rationale for combining tetrahydrocannabinol and cannabidiol. Med Hypotheses 66, 234-246.

5. Friedman D, French JA, Maccarrone M (2019) Safety, efficacy, and mechanisms of action of cannabinoids in neurological disorders. Lancet Neurol 18, 504-512.

6. MacCallum CA, Russo EB (2018) Practical considerations in medical cannabis administration and dosing. Eur J Intern Med 49, 12-19.

7. Pertwee RG (2008) The diverse CB1 and CB2 receptor pharmacology of three plant cannabinoids: $\Delta^{9}$-tetrahydrocannabinol, cannabidiol and $\Delta^{9}$-tetrahydrocannabivarin. $\mathrm{Br} J$ Pharmacol 153, 199-215.

8. Izzo AA, Borrelli F, Capasso R, Di Marzo V, Mechoulam R (2009) Non-psychotropic plant cannabinoids: new therapeutic opportunities from an ancient herb. Trends Pharmacol Sci 30, 515-527.
9. Jadoon KA, Ratcliffe SH, Barrett DA, Thomas EL, Stott C, Bell JD, O'Sullivan SE, Tan GD (2016) Efficacy and safety of cannabidiol and tetrahydrocannabivarin on glycemic and lipid parameters in patients with type 2 diabetes: a randomized, doubleblind, placebo-controlled, parallel group pilot study. Diabetes Care 39, 1777-1786.

10. Sirikantaramas S, Taura F (2017) Cannabinoids: biosynthesis and biotechnological applications. In: Chandra S, Lata H, ElSohly M (eds) Cannabis sativa L.: Botany and Biotechnology, Springer, Cham, Switzerland, pp 183-206.

11. Taura F, Morimoto S, Shoyama Y, Mechoulam R (1995) First direct evidence for the mechanism of $\Delta^{1}$-tetrahydrocannabinolic acid biosynthesis. $J \mathrm{Am}$ Chem Soc 117, 9766-9767.

12. Taura F, Tanaka S, Taguchi C, Fukamizu T, Tanaka H, Shoyama Y, Morimoto S (2009) Characterization of olivetol synthase, a polyketide synthase putatively involved in cannabinoid biosynthetic pathway. FEBS Lett 583, 2061-2066.

13. Gagne SJ, Stout JM, Liu E, Boubakir Z, Clark SM, Page JE (2012) Identification of olivetolic acid cyclase from Cannabis sativa reveals a unique catalytic route to plant polyketides. Proc Natl Acad Sci USA 109, 12811-12816.

14. Morita H, Wong CP, Abe I (2019) How structural subtleties lead to molecular diversity for the type III polyketide synthases. J Biol Chem 294, 15121-15136.

15. Stout JM, Boubakir Z, Ambrose SJ, Purves RW, Page JE (2012) The hexanoyl-CoA precursor for cannabinoid biosynthesis is formed by an acyl-activating enzyme in Cannabis sativa trichomes. Plant $J$ 71, 353-365.

16. Tan Z, Clomburg JM, Gonzalez R (2018) Synthetic pathway for the production of olivetolic acid in Escherichia coli. ACS Synth Biol 7, 1886-1896.

17. Luo X, et al (2019) Complete biosynthesis of cannabinoids and their unnatural analogues in yeast. Nature 567, 123-126.

18. Yang X, et al (2016) Structural basis for olivetolic acid formation by a polyketide cyclase from Cannabis sativa. FEBS J 283, 1088-1106.

19. Fellermeier M, Zenk MH (1998) Prenylation of olivetolate by a hemp transferase yields cannabigerolic acid, the precursor of tetrahydrocannabinol. FEBS Lett 427, 283-285.

20. Fellermeier M, Eisenreich W, Bacher A, Zenk MH (2001) Biosynthesis of cannabinoids. Incorporation experiments with ${ }^{13} \mathrm{C}$-labeled glucoses. Eur J Biochem 268, 1596-1604.

21. Page JE, Boubakir Z (2014) Aromatic prenyltransferase from Cannabis. Patent No. US 8884100 B2.

22. Kuzuyama T, Noel JP, Richard SB (2005) Structural basis for the promiscuous biosynthetic prenylation of aromatic natural products. Nature 435, 983-987. 
23. Zirpel B, Degenhardt F, Martin C, Kayser O, Stehle F (2017) Engineering yeasts as platform organisms for cannabinoid biosynthesis. J Biotechnol 259, 204-212.

24. Qian S, Clomburg JM, Gonzalez R (2019) Engineering Escherichia coli as a platform for the in vivo synthesis of prenylated aromatics. Biotechnol Bioeng 116, 1116-1127.

25. Valliere MA, Korman TP, Woodall NB, Khitrov GA, Taylor RE, Baker D, Bowie JU (2019) A cell-free platform for the prenylation of natural products and application to cannabinoid production. Nat Commun 10, ID 565.

26. Hazekamp A, Choi YH, Verpoorte R (2004) Quantitative analysis of cannabinoids from Cannabis sativa using 1H-NMR. Chem Pharm Bull 52, 718-721.

27. Sirikantaramas S, Morimoto S, Shoyama Y, Ishikawa Y, Wada Y, Shoyama Y, Taura F (2004) The gene controlling marijuana psychoactivity: molecular cloning and heterologous expression of $\Delta^{1}$-tetrahydrocannbinolic acid synthase from Cannabis sativa L. J Biol Chem 279, 39767-39774.

28. Taura F, Sirikantaramas S, Shoyama Y, Yoshikai K, Shoyama Y, Morimoto S (2007) Cannabidiolicacid synthase, the chemotype-determining enzyme in the fiber-type Cannabis sativa. FEBS Lett 581, 2929-2934.

29. Kutchan TM, Dittrich H (1995) Characterization and mechanism of the berberine bridge enzyme, a covalently flavinylated oxidase of benzophenanthridine alkaloid biosynthesis in plants. J Biol Chem 270, 24475-24481.

30. Caputi L, et al (2018) Missing enzymes in the biosynthesis of the anticancer drug vinblastine in Madagascar periwinkle. Science 360, 1235-1239.

31. Iijima $M$, et al (2017) Identification and characterization of daurichromenic acid synthase active in antiHIV biosynthesis. Plant Physiol 174, 2213-2230.

32. Taura F, Dono E, Sirikantaramas S, Yoshimura K, Shoyama Y, Morimoto S (2007) Production of $\Delta^{1}$-tetrahydrocannbinolic acid by the biosynthetic enzyme secreted from transgenic Pichia pastoris. Biochem Biophys Res Commun 361, 675-680.

33. Shoyama Y, et al (2012) Structure and function of $\Delta^{1}$-tetrahydrocannbinolic acid (THCA) synthase, the enzyme controlling the psychoactivity of Cannabis sativa. J Mol Biol 423, 96-105.
34. Sirikantaramas S, Taura F, Tanaka Y, Ishikawa Y, Morimoto S, Shoyama Y (2005) Tetrahydrocannabinolic acid synthase, the enzyme controlling marijuana psychoactivity, is secreted into the storage cavity of the glandular trichomes. Plant Cell Physiol 46, 1578-1582.

35. Morimoto S, Tanaka Y, Sasaki K, Tanaka H, Fukamizu T, Shoyama Y, Shoyama Y, Taura F (2007) Identification and characterization of cannabinoids that induce cell death through mitochondrial permeability transition in Cannabis leaf cells. J Biol Chem 282, 20739-20751.

36. Taura F, Morimoto S, Shoyama Y (1996) Purification and characterization of cannabidiolic-acid synthase from Cannabis sativa L. Biochemical analysis of a novel enzyme that catalyzes the oxidocyclization of cannabigerolic acid to cannabidiolic acid. $J$ Biol Chem 271, 17411-17416.

37. Zirpel B, Kayser O, Stehle F (2018) Elucidation of structure-function relationship of THCA and CBDA synthase from Cannabis sativa L. J Biotechnol 284, 17-26.

38. Laverty KU, Stout JM, Sullivan MJ, Shah H, Gill N, Holbrook L, Deikus G, Sebra R, et al (2019) A physical and genetic map of Cannabis sativa identifies extensive rearrangements at the THC/CBD acid synthase loci. Genome Res 29, 146-156.

39. Zirpel B, Stehle F, Kayser O (2015) Production of $\Delta^{9}$-tetrahydrocannabinolic acid from cannabigerolic acid by whole cells of Pichia (Komagataella) pastoris expressing $\Delta^{9}$-tetrahydrocannabinolic acid synthase from Cannabis sativa L. Biotechnol Lett 37, 1869-1875.

40. Susani M, Zimniak P, Fessl F, Ruis H (1976) Localization of catalase A in vacuoles of Saccharomyces cerevisiae: evidence for the vacuolar nature of isolated yeast peroxisomes. Hoppe Seylers Z Physiol Chem 357, 961-970.

41. Appendino G, Gibbons S, Giana A, Pagani A, Grassi G, Stavri M, Smith E, Rahman MM (2008) Antibacterial cannabinoids from Cannabis sativa: a structureactivity study. $J$ Nat Prod 71, 1427-1430.

42. Amada N, Yamasaki Y, Williams CM, Whalley BJ (2013) Cannabidivarin (CBDV) suppresses pentylenetetrazole (PTZ)-induced increases in epilepsy-related gene expression. PeerJ 1, ID 214. 